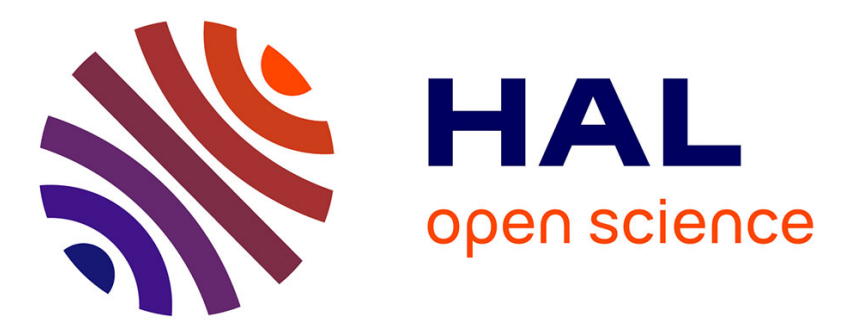

\title{
Complexité de la gestion des médicaments relevant du dispositif pérenne post-ATU dans un centre hospitalier universitaire
}

\author{
A. Bros, F. Reymond, C. Petit, G. Viard-Gaudin, A. Lehmann, L. Foroni, P. \\ Bedouch, C. Chapuis
}

\section{To cite this version:}

A. Bros, F. Reymond, C. Petit, G. Viard-Gaudin, A. Lehmann, et al.. Complexité de la gestion des médicaments relevant du dispositif pérenne post-ATU dans un centre hospitalier universitaire. Le Pharmacien Hospitalier et Clinicien, 2019, 54, pp.126 - 130. 10.1016/j.phclin.2018.09.007 . hal03486283

\section{HAL Id: hal-03486283 https://hal.science/hal-03486283}

Submitted on 20 Dec 2021

HAL is a multi-disciplinary open access archive for the deposit and dissemination of scientific research documents, whether they are published or not. The documents may come from teaching and research institutions in France or abroad, or from public or private research centers.
L'archive ouverte pluridisciplinaire HAL, est destinée au dépôt et à la diffusion de documents scientifiques de niveau recherche, publiés ou non, émanant des établissements d'enseignement et de recherche français ou étrangers, des laboratoires publics ou privés.

\section{(ㄷ)(1) $\$$}

Distributed under a Creative Commons Attribution - NonCommerciall 4.0 International 


\section{Complexité de la gestion des médicaments relevant du dispositif pérenne post-ATU dans un centre hospitalier universitaire}

Complex economic management of "post-temporary authorization" for costly drugs in a

\section{French university hospital}

BROS Aurélie, Pharmacien Assistant, Pôle pharmacie, CHU Grenoble Alpes

REYMOND Fabienne, Pharmacien Praticien Hospitalier, Pôle pharmacie, CHU Grenoble Alpes

PETIT Camille, Interne en pharmacie, Pôle pharmacie, CHU Grenoble Alpes

VIARD-GAUDIN Gwendal, Pharmacien Assistant, Pôle pharmacie, CHU Grenoble Alpes

LEHMANN Audrey, Pharmacien Praticien Hospitalier, Pôle pharmacie, CHU Grenoble Alpes

FORONI Luc, Pharmacien, OMEDIT Auvergne Rhône-Alpes

BEDOUCH Pierrick, Pharmacien PU-PH, Pôle pharmacie, CHU Grenoble Alpes, Université Grenoble Alpes, CNRS, TIMC-IMAG, UMR5525e

CHAPUIS Claire, Pharmacien Praticien Hospitalier, Pôle pharmacie, CHU Grenoble Alpes

Auteur correspondant: Aurélie BROS

Pôle Pharmacie, CHU GRENOBLE ALPES, CS 10217, 38043 Grenoble cedex 9

0476767349

abros@chu-grenoble.fr

Conflits d'intérêts : aucun 


\section{Résumé}

Introduction. - Le mode de financement des spécialités relevant du dispositif pérenne postautorisation temporaire d'utilisation (ATU) et les exigences pour l'obtention du remboursement des spécialités en sus des groupes homogènes de séjours (GHS) évoluent. Cette situation amène les équipes pharmaceutiques en charge de leur gestion à s'adapter. Ainsi, nous nous interrogeons sur les moyens à mettre en œuvre pour assurer celle-ci et son impact.

Matériels et Méthodes. - Une analyse de la gestion des spécialités post-ATU par notre équipe pharmaceutique pour l'année 2016 a été réalisée.

Résultats. - Pour notre CHU, le FICHCOMP Médecine Chirurgie Obstétrique (MCO) de 2016 s'élevait à environ $30270 \mathrm{k€}$ dont $6500 \mathrm{k} €$ ne concernaient que les médicaments postATU, soit environ 21,5\% du budget total des médicaments en sus des GHS. Notre suivi a permis d'identifier de nombreuses prescriptions n'apparaissant pas dans le FICHCOMP et qu'il nous a été possible de rattraper informatiquement $(272,577 \mathrm{k} €)$ après avoir identifié les problématiques.

Discussion/Conclusion. - Le suivi des spécialités incluses dans le dispositif pérenne postATU demande une veille réglementaire quotidienne. Leur remboursement aux établissements de santé implique que ceux-ci procèdent à des déclarations précises et actualisées. De ce fait les changements de statut, de code UCD (unité commune de dispensation) et de prix doivent être renseignés dans les logiciels de prescription en temps réel. Ces activités administratives ne doivent toutefois pas masquer le rôle du pharmacien dans le bon usage de ces spécialités.

Mots-clés : Bon usage; Dispositif pérenne post-ATU; Financement; Remboursement ; Spécialités en sus des GHS 


\begin{abstract}
Introduction. - The means of extending funding for specialties initially covered by a Temporary Use Authorization and the requirements for reimbursement by the French national health insurance system of specialties outside of the diagnosis-related groups (DRG) classification are changing. This situation requires adaptation on the part of pharmaceutical teams in charge of their management. We therefore investigated means of ensuring good management of the change, and its impact.
\end{abstract}

Materials and Methods. - An analysis was made of the management of specialties with “post-Temporary Use Authorization” by our pharmaceutical team in 2016.

Results. - In 2016, in Grenoble University Hospital, the "FICHCOMP" list of non-DRG specialties in obstetric medicine and surgery amounted to approximately $€ 30,270,000$, $€ 6,500,000$ of which concerned only "post-Temporary Use Authorization" drugs: i.e., approximately $21.5 \%$ of the total non-DRG drug budget. Monitoring identified numerous prescriptions not included in the FICHCOMP, which it was possible to into take account in the IT system after identifying the underlying problem, for a total $€ 272,577$.

Discussion/Conclusion. - Monitoring "post-Temporary Use Authorization" specialties requires a daily regulatory watch. For health establishments to receive national health insurance reimbursement requires accurate updated reporting. Therefore, any change in status, CUD (common dispensing unit) code or prices must be entered in real time in the prescription software. These administrative activities should not obscure the role of the pharmacist in the proper use of these specialties.

Keywords: Proper use; post-Temporary Use Authorization; Funding; Reimbursement; NonDRG specialties 


\section{Contexte}

Certains médicaments innovants peuvent faire l'objet d'une autorisation temporaire d'utilisation (ATU). Durant cette période, le laboratoire titulaire des droits d'exploitation du médicament peut fournir ceux-ci aux établissements de santé à titre gracieux ou leur vendre. Le montant est librement fixé par l'industriel et les dépenses des établissements de santé peuvent être prises en charge par une dotation spécifique au titre des missions d'enseignement, de recherche, de référence et d'innovation (MERRI).

Du 1er avril 2012 et au 31 décembre 2013, un dispositif expérimental a été mis en place pour que toute spécialité pharmaceutique ayant fait l'objet d'ATU et qui bénéficie d'une autorisation de mise sur le marché (AMM), puisse être fournie, achetée, utilisée et prise en charge au profit des patients par les collectivités publiques au titre des MERRI [1]. On parle alors de spécialité post-ATU et elle ne peut bénéficier du dispositif post-ATU que si elle figure sur le tableau le tableau intitulé « tableau dispositif pérenne » [1]. Celui-ci précise, pour chaque spécialité, la date de dépôt d'une demande d'inscription au remboursement par le laboratoire, la date limite de prise en charge au titre de ce dispositif (date d'octroi de l'AMM + 7 mois ou date définie par dérogation du Ministre) et enfin la date d'inscription ou de refus d'inscription au remboursement [1]. Le processus de mise à disposition des médicaments innovants est illustré par la Figure 1.

Le tableau dispositif pérenne permet aux établissements de connaître les spécialités médicales concernées par le dispositif pérenne post-ATU et la durée valide de la prise en charge associée à ce dispositif. En 2014, la loi de financement de la Sécurité sociale (LFSS) a institué un dispositif pérenne en relais de ce dispositif expérimental par l'article 48 [2]. Le financement MERRI s'achève soit à la date de publication au Journal Officiel (JO) de 
l'inscription sur la liste des médicaments agrées aux collectivités et autres services sociaux, soit sept mois après la date d'octroi de l'AMM en l'absence de publication de l'agrément aux collectivités au cours de cette période, soit un mois après la date d'octroi de l'AMM en l'absence de réception de dépôt par l'industriel de la demande d'agrément aux collectivités auprès de la Commission de la Transparence. Après publication au JO de l'agrément aux collectivités au titre de l'AMM, la prise en charge hospitalière se fait soit au titre des tarifs des prestations d'hospitalisation afférents aux groupes homogènes de séjour (GHS), soit à titre dérogatoire en sus des prestations d'hospitalisation via l'inscription sur la liste dite « en sus ». Le mode de financement décrit précédemment était en vigueur jusqu'au $1^{\mathrm{er}}$ janvier 2017.

En effet, la LFSS a créé en 2017 un fonds de financement de l'innovation pharmaceutique (FFIP) qui a pour objet de mieux réguler l'impact des dépenses résultant de l'arrivée d'innovations thérapeutiques en les lissant au-delà de l'exercice budgétaire. Ce fonds modifie l'articulation entre le pilotage de l'objectif national de dépenses d'assurance maladie (ONDAM) et les dépenses d'innovations liées à la liste en sus, aux dispositifs d'ATU et postATU et aux médicaments rétrocédés. La CNAMTS a versé en 2017 au FFIP une dotation de 876 millions d'euros pour assurer sa trésorerie initiale. A terme, les dotations annuelles du fonds seront lissées sur la base des dépenses des 5 années écoulées, ce seront les dotations qui seront comptabilisées au sein de chaque ONDAM et non les dépenses annuelles du FFIP [3]. Les modalités de financement des médicaments sous ATU et post-ATU ne dépendent donc plus de la publication des circulaires budgétaires [4] mais sont facturables en sus du GHS. Par conséquent, depuis le $1^{\text {er }}$ janvier 2017, l'administration de ces médicaments au cours d'une hospitalisation donne lieu à un circuit de versement identique à celui mis en place pour le remboursement des produits de la liste en sus, en lieu et place d'un financement par MERRI [5]. 
Depuis 2012, le codage UCD permet d'inscrire l'ensemble des prescriptions des médicaments concernés administrés à l'hôpital à travers l'outil FICHCOMP du programme de médicalisation des systèmes d'information (PMSI). La consommation de tous les médicaments avec AMM (inscrits sur la liste en sus du GHS), ATU ou post-ATU est systématiquement renseignée via FICHCOMP par les établissements de santé prescripteurs (publics ou privés) pour demander le remboursement de ces derniers. Les montants délégués correspondent à l'euro près aux montants déclarés dans FICHCOMP. Ces déclarations sont cumulatives sur l'année. Il est donc de la responsabilité de l'établissement de santé de bien déclarer ses propres consommations afin de bénéficier d'un financement adéquat. La prise en charge dure jusqu'à ce qu'une décision relative à l'inscription de ce médicament, au titre de son AMM, sur une des listes (in GHS ou en sus du GHS) et, le cas échéant, jusqu'à ce que l'avis de fixation du tarif de responsabilité ou du prix ait été publié par le Comité économique des produits de santé (CEPS). Le prix du médicament post-ATU reste celui de l'ATU jusqu'à la fixation d'un prix. Le remboursement post-ATU n'est appliqué que dans les indication(s) de l'ATU ou si une dérogation spécifique et exceptionnelle est accordée par la Haute autorité de santé (HAS).

Enfin, le respect de l'engagement tenu par les établissements de santé envers le contrat d'amélioration de la qualité et de l'efficience des soins (CAQES) permet un remboursement à $100 \%$ des produits en sus par l'Assurance-maladie. Une organisation pour la gestion de ces médicaments est donc indispensable dans un centre hospitalier universitaire. Ainsi, nous nous interrogeons sur les moyens à mettre en œuvre pour assurer celle-ci et son impact.

\section{II.Matériel et méthodes}

Notre CHU est un établissement de 2158 lits ayant une activité de médecine, chirurgie et obstétrique $(\mathrm{MCO})$, de soins de suite et de réadaptation (SSR) et d'hospitalisation à 
domicile (HAD) assurant toutes les disciplines médicales à l'exception de la prise en charge des grands brûlés. Actuellement, l'équipe pharmaceutique dédiée à la gestion du FICHCOMP et participant quotidiennement au contrat de bon usage (CBU) implique des pharmaciens hospitaliers, des internes en pharmacie, des externes en pharmacie et des préparateurs en pharmacie. Cette équipe assure une mise à jour régulière des référentiels de bon usage et une validation des prescriptions hors-référentiels en collaboration avec les équipes médicales. L'équipe pharmaceutique assure également le suivi mensuel des dispensations (voire quotidien pour certains médicaments) et surtout la traçabilité informatique de l'administration des médicaments en sus. Cela permet d'identifier un nombre conséquent d'administrations n'apparaissant pas dans le FICHCOMP (séjours en erreur, erreurs de prescription, oublis de validation d'administration, prescriptions hors-référentiel, etc..) et de permettre le rattrapage informatique de celles-ci.

Une analyse de la gestion des spécialités post-ATU par notre équipe pharmaceutique pour l'année 2016 a été réalisée. Pour cela, nous avons récupéré les montants déclarés dans le FICHCOMP et ceux accordés en remboursement sur la plateforme e-PMSI pour chaque spécialité. Nous avons évalué le montant des rattrapages informatiques assurés par l'équipe pharmaceutique après avoir identifié les difficultés rencontrées pour obtenir leur remboursement. Par ailleurs, nous avons calculé le délai moyen entre la date d'entrée dans le dispositif pérenne post-ATU et la date d'inscription au remboursement pour l'ensemble des spécialités du tableau dispositif pérenne [1]. Le délai théorique correspond à la date d'octroi de l'AMM + 7 mois

\section{III.Résultats}

Depuis 2006, date de la signature du premier CBU, et jusqu'en 2015, notre CHU a obtenu chaque année le remboursement à 100\% des médicaments et des dispositifs médicaux 
en sus des GHS. Toutefois, l'augmentation des dépenses pour les médicaments en sus au CHU (+17\% en 5 ans), comme dans les autres CHU, nécessite un suivi plus conséquent du respect des référentiels de bon usage et des déclarations via le FICHCOMP.

Le suivi des médicaments relevant du dispositif pérenne post-ATU est d'ailleurs de plus en plus conséquent et complexe, si bien qu'en 2016, 20 spécialités étaient inscrites sur la liste des médicaments relevant du dispositif pérenne post-ATU [1]. En pratique, le dispositif dure en moyenne 13,4 mois $[7,7 ; 19,1]$. Pour rappel, le délai théorique correspond à la date d'octroi de l'AMM + 7 mois [1]. Dans notre CHU en 2016, 11 spécialités étaient régulièrement prescrites et suivies : daratumumab (Darzalex®), sébélipase alfa (Kanuma®), mépolizumab (Nucala®), nivolumab (Opdivo®), ivacaftor/lumacaftor (Orkambi®), sélexipag (Uptravi®), blinatumomab (Blincyto®), vedolizumab (Entyvio®), pembrolizumab (Keytruda $\left.{ }^{\circledR}\right)$, osimertinib (Tagrisso®) et dexaméthasone (Neofordex $\left.{ }^{\circledR}\right)$. Le tableau I présente le bilan économique de 9 médicaments sur 11 relevant du dispositif pérenne post-ATU en 2016 et suivies dans notre CHU. L'osimertinib (Tagrisso®) et la dexaméthasone (Neofordex $\left.{ }^{\circledR}\right)$ n'apparaissent pas dans le tableau I car ne représentaient pas un enjeu économique important comparativement aux autres produits.

Ces médicaments nécessitent un suivi spécifique par l'équipe pharmaceutique en raison de leur impact budgétaire. En effet, le FICHCOMP MCO de 2016 s'élevait à environ 30270 k€ dont $6500 \mathrm{k} €$ ne concernaient que les médicaments post-ATU, soit environ 21,5\% du budget total des médicaments en sus du GHS. De plus, il a fallu constamment interchanger les différents codes UCD existants pour chaque spécialité en fonction des dates de fin du dispositif pérenne post-ATU, afin que ces médicaments puissent être pris en compte et remboursés. D'autant plus que certaines spécialités disposent d'indications dans le cadre de l'AMM et d'autres indications dans le cadre du post-ATU (nivolumab Opdivo® par exemple). 
Par ailleurs, notre suivi a permis d'identifier de nombreuses prescriptions n'apparaissant pas dans le FICHCOMP et qu'il nous a été possible de rattraper informatiquement. Le montant de ces rattrapages s'élève à 272,577 k€ en 2016, sans compter les rattrapages quotidiens du préparateur en pharmacie et des étudiants en pharmacie qui sont chargés de suivre nominativement les administrations de chaque unité dispensée aux unités de soins pour certaines spécialités et que nous ne traçons pas. Ce suivi spécifique nous permet de limiter les écarts entre les unités dispensées et les unités administrées.

\section{IV.Discussion - Conclusion}

Dans la gestion des financements des médicaments administrés à l'hôpital, le dispositif français des ATU est atypique par rapport aux modèles étrangers, mais doit être préservé. Il permet un accès large et rapide de patients atteints de maladies graves, sans alternative thérapeutique, à de nouveaux médicaments, sans attendre l'AMM et l'aboutissement du long processus de fixation des prix, plusieurs mois voire plusieurs années avant que ces médicaments soient accessibles dans d'autres pays. En Allemagne, les médicaments sont disponibles dès l'obtention de l'AMM, sur la base d'un prix librement fixé par les industriels. La procédure d'évaluation en vue de la détermination du prix et de l'admission au remboursement intervient dans un délai d'un an suivant cette mise sur le marché et se déroule parallèlement à celle-ci. Au Royaume-Uni, l'évaluation initiale se fait de manière anticipée, avant l'AMM, avec un financement et un accès temporaires pour les patients dans l'attente de l'évaluation finale. Elle se fonde notamment sur le rapport coût-efficacité du médicament ; elle conduit à une recommandation de prise en charge par le National Health Service (NHS), le cas échéant avec restrictions, ou à un refus, le cas échéant temporaire, de prise en charge (dans ce dernier cas le NHS n'a aucune obligation de mettre à disposition le produit pour les patients). En Italie, pour les médicaments classés comme remboursables par l'Agenzia Italiana 
del Farmaco (AIFA), la fixation du prix est déterminée après négociation avec le laboratoire. Depuis 2011, l'AIFA a fixé un prix de remboursement maximum pour les médicaments remboursables. Ce «cap » est basé sur une enquête des prix en cours dans les pays de l'Union Européenne. Le recours à des contrats de performance ou de partage de risques est particulièrement développé pour les médicaments onéreux, avec un accent sur le suivi en vie réelle via des registres nationaux. Plus récemment, un mécanisme de paiement à la performance a été testé, le système de santé italien payant le traitement à l'industriel ex post pour les seuls patients répondeurs.

Cependant, si les modèles étrangers sont utiles à la réflexion, il est difficile d'envisager toute transposition tant les organisations des systèmes de santé et leur mode de régulation diffèrent $[6,7]$.

En France, le dispositif des ATU et post-ATU ne peut fonctionner sans la participation active des établissements de santé en ce sens et une perpétuelle réadaptation des pratiques. Une activité pharmaceutique à temps plein pour le bon usage et la gestion économique des médicaments sous ATU, relevant du dispositif pérenne post-ATU et en sus du GHS dans un CHU est indispensable et n'est plus à justifier. Les pharmaciens doivent développer une collaboration avec le contrôle de gestion pour pouvoir se concentrer sur le bon usage du médicament au sens strict du terme et n'intervenir que sur les tâches présentant une plus-value pharmaceutique (expertise sur les médicaments, relations et échanges avec les médecins prescripteurs). Les nouvelles exigences imposées par l'Assurance-maladie viennent d'ailleurs complexifier cette activité.

Le délai théorique d'inscription sur la liste en sus du GHS et donc de remboursement de la spécialité au titre de son AMM (date d'octroi de l'AMM +7 mois) est en pratique doublé (13,4 mois en moyenne). Cette donnée est confirmée dans le rapport d'information du Sénat du 18 juin 2018 [8]. Ce délai entraine un suivi plus long par l'équipe pharmaceutique de 
spécialités pour lesquelles nous rencontrons parfois des difficultés pour obtenir le remboursement (tableau I). Par ailleurs, les inscriptions/radiations au JO selon les indications AMM et non plus par médicament (voriconazole Vfend ${ }^{\circledR}$ injectable par exemple), nous imposent de suivre individuellement l'indication de chaque prescription pour nous assurer que le remboursement du médicament nous est autorisé. Le cas échéant, nous sommes dans l'obligation de retirer les déclarations du FICHCOMP. Pour limiter ces situations, un travail important d'information et d'échange avec les prescripteurs a été et sera nécessaire. Le logiciel d'aide à la prescription est un outil indispensable pour identifier les prescriptions hors référentiels et/ou non remboursées en sus mais n'est actuellement pas suffisamment performant pour gérer au mieux ces nouvelles exigences de suivi de prescriptions à l'indication.

Par ailleurs, en 2016, l'absence de compensation à l'euro a été constatée par l'ensemble des CHU dans le cadre des notifications de crédits réceptionnées à l'issue de la première circulaire de campagne 2017. En effet, après rapprochement par le CHRU de Poitiers des délégations de crédits successives avec les données des FICHCOMP 2016 des CHU, il ressort clairement un sous-financement supérieur à 12 M€ sur un total de dépenses rattachées aux séjours valorisés en 2016 de 245 M€, ce qui correspond à un taux de prise en charge de 95\% (courrier de Jean-Pierre Dewitte à Cécile Courreges, Directrice Générale de l'Offre de Soins).

Il serait intéressant de réaliser un état des lieux national concernant l'organisation de cette activité dans l'ensemble des CHU français et ainsi mettre en place des actions pérennes pour accompagner l'équipe pharmaceutique, notamment sur le plan organisationnel de rendu du FICHCOMP. 


\section{$\underline{\text { Références : }}$}

1. Ministère des Solidarités et de la Santé. Autorisations temporaires d'utilisation (ATU) [Internet]. 2017. Disponible sur : http://solidarites-sante.gouv.fr/soins-etmaladies/medicaments/professionnels-de-sante/autorisation-de-mise-sur-lemarche/article/autorisations-temporaires-d-utilisation-atu. Accédé le 27/08/2018

2. Article 48 de la loi 2013 - 1203 du 23 décembre 2013 de financement de la sécurité sociale pour 2014 - article L. 162 - 16 - 5 - 2 du code de la sécurité sociale. Code de la sécurité sociale. Accédé le 27/08/2018

3. Projet de loi de financement de la sécurité sociale pour 2017 - XIVe législature Assemblée nationale [Internet]. 2016. Disponible sur : http://www2.assembleenationale.fr/documents/notice/14/projets/p14072/(index)/depots. Accédé le 27/08/2018

4. Fédération de l'Hospitalisation Privée. Modification du Financement des médicaments sous ATU et Post ATU au 1er Janvier 2017 [Internet]. Fédération de l'Hospitalisation Privée. 2017. Disponible sur : http://www.fhpmco.fr/2017/01/24/modification-financementmedicaments-atu-post-atu-1er-janvier-2017/. Accédé le 27/08/2018

5. Fédération de l'Hospitalisation Privée. Fiche d'Information - Pharmacie sous Autorisation Temporaire d'Utilisation [Internet]. 2017. Disponible sur : http://www.fhpmco.fr/wp-content/uploads/2017/03/FI-PHX.pdf. Accédé le 27/08/2018

6. Joubert S. Accélération de l'accès à l'innovation pharmaceutique : état des lieux et perspectives. Mémoire de DES de Pharmacie, 2015, Université d'Angers. [Internet]. Disponible sur : angers.fr/fichiers/20061382/2015PPHA5045/fichier/5045F.pdf. Accédé le 27/08/2018

7. Innovation médicamenteuse en cancérologie - Étude internationale sur la définition et l'accès à l'innovation. INCa, janvier 2018 [Internet]. Disponible sur : http://www.ecancer.fr/Expertises-et-publications/Catalogue-des-publications/Innovation-medicamenteuseen-cancerologie-Etude-internationale-sur-la-definition-et-1-acces-a-l-innovation. Accédé le 27/08/2018

8. Daudigny Y, Deroche C, Guillotin V. Médicaments innovants : consolider le modèle français d'accès précoce. Rapport d'information $n^{\circ} 569$ (2017-2018) fait au nom de la mission d'évaluation et de contrôle de la sécurité sociale et de la commission des affaires sociales, Sénat, juin 2018. [Internet]. Disponible sur : http://www.senat.fr/rap/r17-569/r17-569.html. Accédé le 27/08/2018 


\section{Légendes des figure - tableau}

Figure and Table legends

Tableau 1: Bilan économique des spécialités relevant du dispositif pérenne post-ATU en 2016 (source e-PMSI)

Table I: Financial assessment of "post-Temporary Use Authorization” drugs

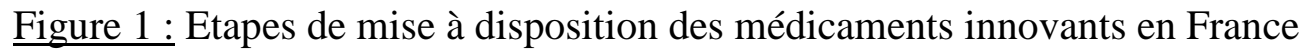

Figure 1: Steps for provision of innovative medicines in France 


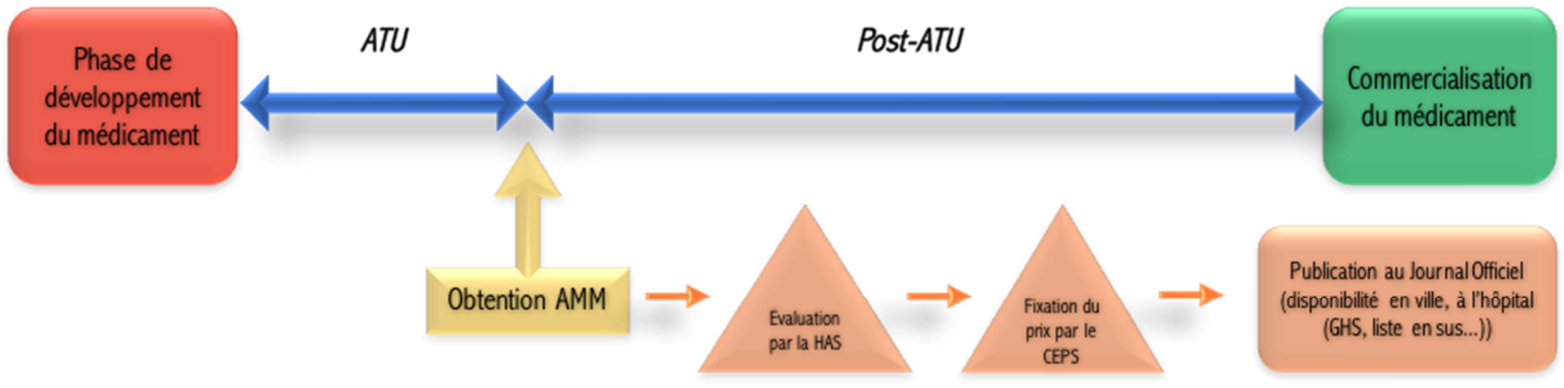




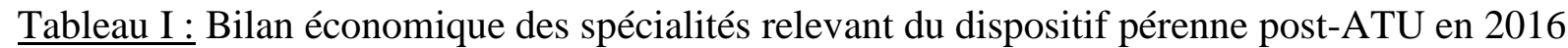
(source e-PMSI)

\begin{tabular}{|c|c|c|c|c|}
\hline Spécialités & $\begin{array}{l}\text { Montants } \\
\text { FICHCOMP }\end{array}$ & $\begin{array}{l}\text { Rattrapages par } \\
\text { l'équipe CBU }\end{array}$ & $\begin{array}{c}\text { Remboursements } \\
\text { accordés }\end{array}$ & $\begin{array}{l}\text { Difficultés rencontrées pour } \\
\text { obtenir le remboursement }\end{array}$ \\
\hline $\begin{array}{l}\text { Daratumumab } \\
\text { (Darzalex }()\end{array}$ & 356823 euros & 177006 euros & $100 \%$ & $\begin{array}{c}5 \text { codes UCD différents pour } 2 \\
\text { dosages disponibles }\end{array}$ \\
\hline $\begin{array}{l}\text { Sébélipase alfa } \\
\left.\text { (Kanuma }{ }^{\circledR}\right)\end{array}$ & 210877 euros & & $100 \%$ & \\
\hline $\begin{array}{l}\text { Mépolizumab } \\
\left.\text { (Nucala }{ }^{\circledR}\right)\end{array}$ & 179867 euros & & $100 \%$ & \\
\hline Nivolumab (Opdivo®) & 2689464 euros & & $100 \%$ & $\begin{array}{l}\text { AMM obtenue en novembre } \\
2016 \text { donc } 2 \text { UCD valides } \\
\text { (celle de l'ATU et celle de } \\
\text { l'AMM) }\end{array}$ \\
\hline $\begin{array}{c}\text { Ivacaftor/lumacaftor } \\
\text { (Orkambi®) }\end{array}$ & 31506 euros & 237 euros & $100 \%$ & \\
\hline Sélexipag (Uptravi®) & 8917 euros & & $100 \%$ & \\
\hline $\begin{array}{l}\text { Blinatumomab } \\
\text { (Blincyto@) }\end{array}$ & 126957 euros & 95334 euros & $100 \%$ & $\begin{array}{l}\text { Code UCD rejeté durant les } \\
\text { premiers mois du dispositif }\end{array}$ \\
\hline $\begin{array}{l}\text { Vedolizumab } \\
\text { (Entyvio®) }\end{array}$ & 1468606 euros & & $100 \%$ & \\
\hline $\begin{array}{l}\text { Pembrolizumab } \\
\left.\text { (Keytruda }{ }^{\circledR}\right)\end{array}$ & 1674078 euros & & $100 \%$ & \\
\hline
\end{tabular}

